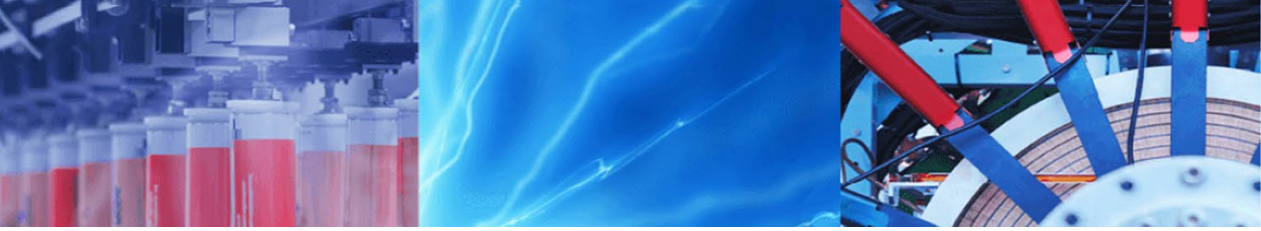

Research Article

\title{
Dynamics of interaction between solitary and rogue wave of the space-time fractional Broer-Kaup models arising in shallow water of harbor and coastal zone
}

\author{
Selina Akter ${ }^{1,2} \cdot$ Ratan Kumar Sen ${ }^{1} \cdot$ Harun-Or- Roshid ${ }^{1}$
}

Received: 26 May 2020 / Accepted: 28 October 2020 / Published online: 16 November 2020

(c) Springer Nature Switzerland AG 2020

\begin{abstract}
Under inquisition, we consider the space-time fractional Broer-Kaup model that replicate the real coastal profile to describe bidirectional wave transmission of long waves in shallow water. We integrate of the model via the unified scheme to derive exact solitary wave solutions. The achieved results present the hyperbolic, trigonometric, and rational functions solutions. All of these solutions convey the periodic and solitonic natures. In particular, we present combo waves that imply a curvy periodic waves in which rogue waves occur in both sides of each waves. The dynamics of obtained nonlinear wave solutions are demonstrated in 3-D and 2-D shapes with definite parametric values. It is shown that the obtained solutions of the model pact a very rich dynamical behavior and can be used to describe seismic type nonlinear wave motion in shallow water waves of the coastal and harbor region.
\end{abstract}

Keywords The space-time fractional Broer-Kaup equation · The unified method · Conformable derivative · Traveling wave solution

\section{Introduction}

Generally, overall the world, configurations in the low lying area of coastal region are often affected by Tsunami wave pressure or cyclonic storm surge and such massive waves of the ocean and consequently damages. Mostly broken tsunami wave moves inland in the form of turbulent hydraulic bores [1]. During tsunami type seismic occur, region is severely damaged. Tsunamis type massive waves create dissipated energy that travels through water medium and when the depth of the ocean decreases, the wave height increase to higher. The velocity of tsunami waves depends on ocean intensity instead of the gap from the source of the wave [2]. It is, therefore, an important event whose study will contribute to better understanding of coastal hazards from seismic waves. Right now, such massive wave related research for the most part focuses on numerical modeling of wave propagation [3-5]. Nonlinear fractional derivatives are the appropriate tools for modeling highly non-linear problems including seismic waves in harbor and coastal region. One of this model is the space-time fractional Broer-Kaup model (FBKM) [3].

In this article, we shed light on the space-time fractional Broer-Kaup model (FBKM) which is significant model to describe bidirectional wave transmission of long waves in shallow water. The usual form of the space-time FBKM [3] is as follows

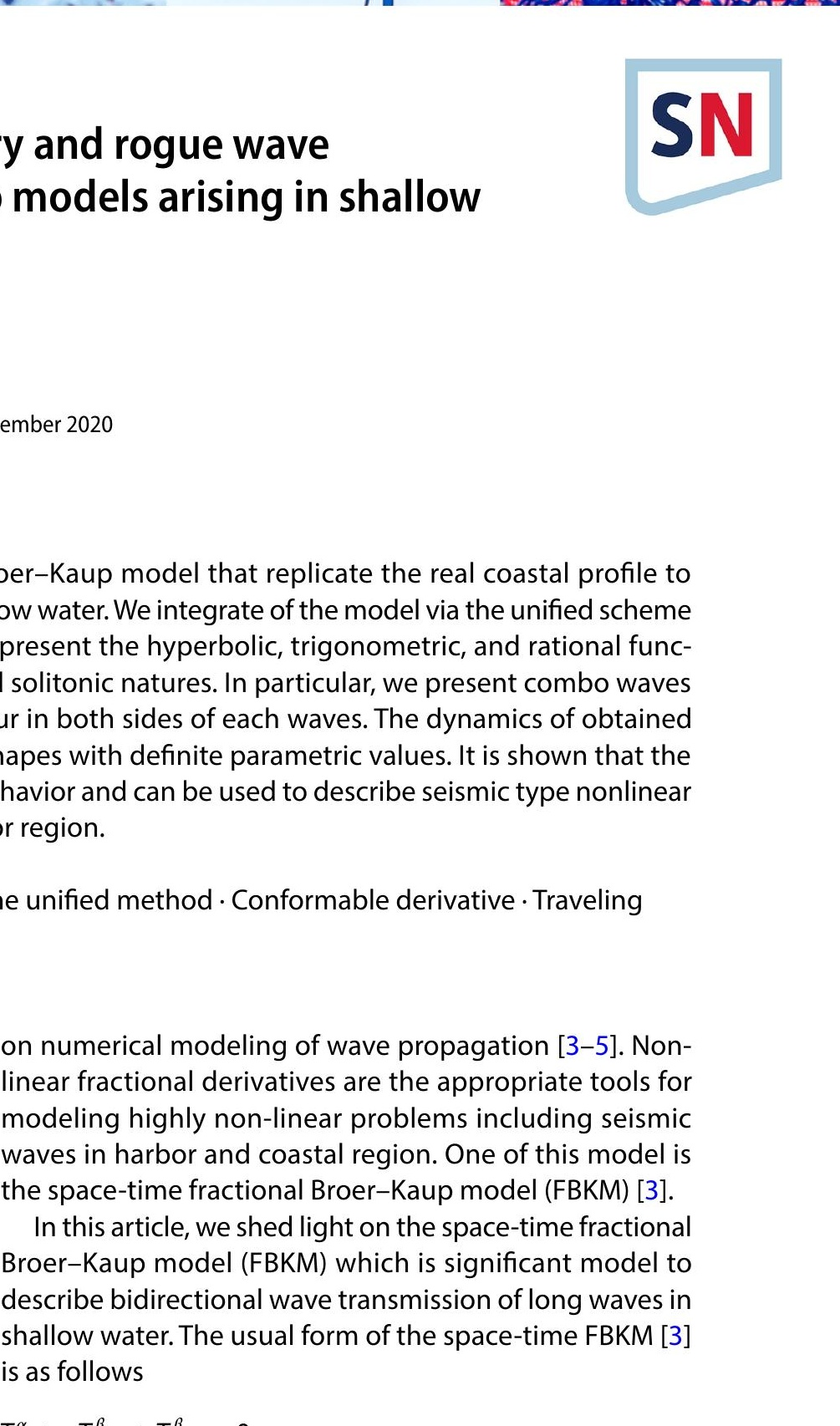

$T_{t}^{\alpha}+u T_{x}^{\beta} u+T_{x}^{\beta} v=0$

$T_{t}^{\alpha} v+T_{x}^{\beta} u+T_{x}^{\beta}(u v)+T_{x}^{\beta} T_{x}^{\beta} T_{x}^{\beta} u=0, \quad t>0,0<\alpha, \beta \leq 1$.

$\triangle$ Harun-Or-Roshid, harunorroshidmd@gmail.com; harun_math@pust.ac.bd| ${ }^{1}$ Department of Mathematics, Pabna University of Science and Technology, 6600, Pabna, Bangladesh. 2Department of Applied Mathematics, Gono Bishwabidyalay, Savar, Dhaka, Bangladesh. 
Here $T_{t}^{\alpha}$ and $T_{x}^{\beta}$ indicate conformable fractional derivative [4] reverence to $t$ and $x$, respectively. Besides, the seismic (earthquake, tsunami and typhoon) waves are reflect as the solitary waves. Solitons are stable and confined nonlinear wave progressed balancing between nonlinearity and dispersion in an arrangement. Due to their rising in a massive diversity in media, extending from fluids $[5,6]$ and astrophysical plasmas $[7,8]$ to semiconductors $[9,10]$ and nonlinear optics $[11,12]$, solitons have converted a sensible purpose for technical exploration. Such hip in natural structures, solitons can similarly be formed in laboratories as dual plasma schemes [13, 14], Joseph-son junctions [15, $16]$ and so on. With this point of view, deriving soliton and its interaction solution of nonlinear wave models attract a much interest of young scientist [17-19]. Currently, many scientists are working with considerable efforts on the fractional differential models as it gives the real properties of natural phenomena [20, 21]. Tozar et. al. [22], Kurt [23], Kurt et. al. [24], Tasbozan [25] and Tasbozan et. al. [26] investigated new solutions for the time fractional integrable dispersive model that arises in ocean engineering, the fractional Bogoyavlensky-Konopelchenko model that arising in fluid dynamics, the fractional coupled Burgers' equation that arises as a model of poly-dispersive sedimentation, the time fractional Benjamin-Ono model that arises internal waves in deep water and the fractional Drinfeld-Sokolov-Wilson system in shallow water waves respectively. Iyiola et. al. [27] studied on the analytical solutions of the system of conformable time-fractional Robertson equations with 1-D diffusion. Cenesiz et. al. [28] established new exact solutions of Burgers' type equations with conformable derivative.

Various approaches are used to study the above fractional models. Recently, Gozukizil et. al. [29] proposed a unified scheme that receiving much attention of young researcher to handle non-linear fractional differential models. Beside this, some researcher has compared the all tanh methods [8, 9] with the unified method [29]. Akcagil and Aydemir [30] have exemplified that the unified method is not only more general than the family of tanh function methods but also it gives many more general solutions than the new members of the family of $\left(G^{\prime} / G\right)$-expansion method. They come to verdict that the unified method gives many more general solutions in an elegant way than the family of the tanh-function methods.

Therefore, the aim of this paper is to solve the spacetime fractional Broer-Kaup equation for finding exact solitary wave solutions via the effective unified method $[29,30]$. This method produces combo waves that imply a curvy periodic waves in which rogue waves occur in both sides of each waves and express in terms of rational, trigonometric and hyperbolic functions.

The structure of this paper goes along with: In Sect. 2, we have recalled the unified method. In Sect. 3, we have presented the exact solutions to the space-time fractional Broer-Kaup equations employing the unified method. In Sect. 4, we have discussed obtaining solutions with the graphical representation and in the last section, conclusions are given.

\section{The unified method}

Here, we illustrate the unified method $[29,30]$ for governing exact solutions of nonlinear partial differential equations (NLPDEs) in the subsequent way. Adopt a NPDE in two independent variables $x$ and $t$ is given by

$P\left(u, u_{x}, u_{x x t}, u_{x x x}, u_{t}, u_{t t}, \ldots\right)=0$,

where $p$ is a polynomial in $u=u(x, t)$ and its several derivatives including the highest order derivatives and nonlinear terms.

Step-1: To convert the NLPDE into Ordinary differential equation $(\mathrm{ODE})$, we operate the traveling wave variable

$u(x, t)=U(\xi), \quad \xi=k x-\omega t$,

where $k$ is constant and $\omega$ is the wave velocity. Now switching (3) into (2) we attain an ODE

$R\left(u_{\xi}, u_{\xi \xi \xi}, u_{\xi \xi}, u u_{\xi} u_{\xi \xi \xi}, \ldots\right)=0$.

Step-2: Now we integrate (4) as many times as possible. Keep the integrating constant to zero.

Assume that the solution of ODE (4) takes the following form

$U(\xi)=I_{0}+\sum_{i=1}^{N}\left[I_{i} S(\xi)^{i}+m_{i} S(\xi)^{-i}\right]$

whose $I_{i}(i=0,1,2, \ldots, N)$ and $m_{i}(i=0,1,2, \ldots, N)$ are constants to be calculated subsequently on the situation that $I_{N}$ and $m_{N}$ cannot be zero at a time. The function $S(\zeta)$ satisfies the Riccati equation,

$S^{\prime}=(S(\xi))^{2}+\lambda$,

whose solutions are specified as follows:

Case-1: Hyperbolic function (when $\lambda<0$ ): 


$$
S(\xi)=\left\{\begin{array}{l}
\frac{\sqrt{-\left(I^{2}+d^{2}\right) \lambda}-I \sqrt{-\lambda} \cosh (2 \sqrt{-\lambda}(\xi+E))}{I \sinh (2 \sqrt{-\lambda}(\xi+E))+d}, \\
\frac{-\sqrt{-\left(I^{2}+d^{2}\right) \lambda}-I \sqrt{-\lambda} \cosh (2 \sqrt{-\lambda}(\xi+E))}{I \sinh (2 \sqrt{-\lambda}(\xi+E))+d}, \\
\frac{\sqrt{-\lambda}\{\cosh (2 \sqrt{-\lambda}(\xi+E))-\sinh (2 \sqrt{-\lambda}(\xi+E))-I\}}{I+\cosh (2 \sqrt{-\lambda}(\xi+E))-\sinh (2 \sqrt{-\lambda}(\xi+E))}, \\
\frac{\sqrt{-\lambda}\{1-\cosh (2 \sqrt{-\lambda}(\xi+E))-\sinh (2 \sqrt{-\lambda}(\xi+E))\}}{I+\cosh (2 \sqrt{-\lambda}(\xi+E))+\sinh (2 \sqrt{-\lambda}(\xi+E))},
\end{array}\right.
$$

Case-2: Trigonometric function (when $\lambda>0$ ):

$$
S(\xi)=\left\{\begin{array}{l}
\frac{\sqrt{\left(I^{2}-d^{2}\right) \lambda}-I \sqrt{\lambda} \cos (2 \sqrt{\lambda}(\xi+E))}{I \sin (2 \sqrt{\lambda}(\xi+E))+d}, \\
\frac{-\sqrt{\left(1^{2}-d^{2}\right) \lambda}-I \sqrt{\lambda} \cos (2 \sqrt{\lambda}(\xi+E))}{I \sin (2 \sqrt{\lambda}(\xi+E))+d}, \\
\frac{\sqrt{\lambda}\{i \cos (2 \sqrt{\lambda}(\xi+E))+\sin (2 \sqrt{\lambda}(\xi+E))-i l\}}{I+\cos (2 \sqrt{\lambda}(\xi+E))-i \sin (2 \sqrt{\lambda}(\xi+E))}, \\
\frac{\sqrt{\lambda}\{-i \cos (2 \sqrt{\lambda}(\xi+E))+\sin (2 \sqrt{\lambda}(\xi+E))+i l\}}{1+\cos (2 \sqrt{\lambda}(\xi+E))+i \sin (2 \sqrt{\lambda}(\xi+E))},
\end{array}\right.
$$

where $I \neq 0$ and $d, E$ are real arbitrary constant.

Case-3: Rational function solutions (when $\lambda=0$ )

$S(\xi)=-\frac{1}{\xi+E}$.

Step-03: By homogeneous balance principle, we determine the positive integer $N$ of (4). Inserting (5) into (4) making use of (6), and then extracting all terms of like powers of $S(\zeta)$ to zero yields an over-determined system of algebraic equations. Solving this algebraic equations for $l_{i}(i=0,1,2, \ldots, N)$ and $m_{i}(i=0,1,2, \ldots, N) k, \omega$ and inserting the values into (5) together with the general solutions of (6), provides us the required solutions of (2).

\section{Solutions for the space-time FBK model via the unified method}

Now, we apply the unified integral scheme to extract exact solutions of (1). First, we consider the following transformation to convert (1) into ordinary differential equation (ODE):

$u(x, t)=U(\xi) ; \quad \xi=a \frac{t^{\alpha}}{\alpha}+b \frac{x^{\beta}}{\beta}$

where $a, b$ are constants.

Plugging (7) into (1), we have the following ODE

$a U^{\prime}+b U U^{\prime}+b V^{\prime}=0$.

$a V^{\prime}+b U^{\prime}+b\left(U V^{\prime}+V U^{\prime}\right)+b^{3} U^{\prime \prime \prime}=0$.

One time integration of (8) with respect to $\xi$, yields

$V=\frac{C_{1}}{b}-\frac{U^{2}}{2}-\frac{a}{b} U$.

Switching (8) into (7) yields a unique equation

$b^{3} U^{\prime \prime}-\frac{b}{2} U^{3}-\frac{3 a}{2} U^{2}+\left(C_{1}+b-\frac{a^{2}}{b}\right) U-C_{2}=0$

here $C_{1}$ and $C_{2}$ are integration constants.

Now balancing the terms $U^{\prime \prime}$ and $U^{3}$ of (11), unknown $N$ becomes $N=1$.

So the trial solution (5) takes the form

$U(\xi)=I_{0}+I_{1} S(\xi)+m_{1} S^{-1}(\xi)$.

Combining (6), (11) and (12) and collecting the coefficients of $S(\xi)$, gives the following set of algebraic equations:

$2 b^{3} I_{1}-\frac{1}{2} b l_{1}^{3}=0 ; \quad-\frac{3}{2} b I_{0} l_{1}^{2}-\frac{3}{2} a l_{1}^{2}=0 ;$

$2 b^{3} I_{1} \lambda-\frac{1}{2} b\left(m_{1} I_{1}^{2}+2 I_{0}^{2} I_{1}+I_{1}\left(I_{0}^{2}+2 I_{1} m_{1}\right)\right)-3 a I_{0} I_{1}-\frac{\left(a^{2}-\overline{b^{2}-b C_{1}}\right) I_{1}}{b}=0 ;$

$-\frac{1}{2} b\left(4 m_{1} I_{0} I_{1}+I_{0}\left(I_{0}^{2}+2 I_{1} m_{1}\right)\right)-\frac{3}{2} a\left(I_{0}^{2}+2 I_{1} m_{1}\right)-\frac{\left(a^{2}-b^{2}-b C_{1}\right) I_{0}}{b}-C_{2}=0 ;$

$2 b^{3} m_{1} \lambda+\frac{1}{2} b\left(m_{1}\left(I_{0}^{2}+2 I_{1} m_{1}\right)+2 l_{0}^{2} m_{1}+I_{1} m_{1}^{2}\right)-3 a m_{1} I_{0}-\frac{\left(a^{2}-b^{2}-b C_{1}\right) m_{1}}{b}=0 ;$ 
$-\frac{3}{2} b m_{1}^{2} I_{0}-\frac{3}{2} a m_{1}^{2}=0 ; \quad 2 b^{3} m_{1} \lambda^{2}-\frac{1}{2} b m_{1}^{3}=0 ;$

Solving the above algebraic equations, we obtain the following sets of constraints:

Set-1:

$C_{1}=-\frac{1}{2} \frac{4 b^{4} \lambda+a^{2}+2 b^{2}}{b}, \quad C_{2}=-\frac{1}{2 b}\left(-\frac{\left(4 b^{4} \lambda+a^{2}+2 b^{2}\right) a}{b}+\frac{2 a^{3}}{b}-\frac{\left(2 a^{2}-2 b^{2}\right) a}{b}\right), \quad l_{0}=-\frac{a}{b}, \quad l_{1}=2 b, \quad m_{1}=0$.

\section{Set-2:}

$C_{1}=-\frac{1}{2} \frac{4 b^{4} \lambda+a^{2}+2 b^{2}}{b}, \quad C_{2}=-\frac{1}{2 b}\left(-\frac{\left(4 b^{4} \lambda+a^{2}+2 b^{2}\right) a}{b}+\frac{2 a^{3}}{b}-\frac{\left(2 a^{2}-2 b^{2}\right) a}{b}\right), \quad I_{0}=-\frac{a}{b}, \quad l_{1}=-2 b, \quad m_{1}=0$

Set-3:

$C_{1}=-\frac{1}{2} \frac{4 b^{4} \lambda+a^{2}+2 b^{2}}{b}, \quad C_{2}=-\frac{1}{2 b}\left(-\frac{\left(4 b^{4} \lambda+a^{2}+2 b^{2}\right) a}{b}+\frac{2 a^{3}}{b}-\frac{\left(2 a^{2}-2 b^{2}\right) a}{b}\right), \quad I_{0}=-\frac{a}{b}, \quad I_{1}=0, \quad m_{1}=2 b$

Set-4:

$C_{1}=-\frac{1}{2} \frac{4 b^{4} \lambda+a^{2}+2 b^{2}}{b}, \quad C_{2}=-\frac{1}{2 b}\left(-\frac{\left(4 b^{4} \lambda+a^{2}+2 b^{2}\right) a}{b}+\frac{2 a^{3}}{b}-\frac{\left(2 a^{2}-2 b^{2}\right) a}{b}\right), \quad I_{0}=-\frac{a}{b}, \quad l_{1}=0, \quad m_{1}=-2 b \lambda$

\section{Set-5:}

$C_{1}=-\frac{1}{2} \frac{-8 b^{4} \lambda+a^{2}+2 b^{2}}{b}, \quad C_{2}=-\frac{1}{2 b}\left(-\frac{\left(-8 b^{4} \lambda+a^{2}+2 b^{2}\right) a}{b}+\frac{2 a^{3}}{b}-\frac{\left(24 b^{4} \lambda+2 a^{2}-2 b^{2}\right) a}{b}+24 b^{3} a \lambda\right), \quad I_{0}=-\frac{a}{b}, \quad I_{1}=2 b, \quad m_{1}=2 b \lambda$

\section{Set-6:}

$$
\begin{aligned}
& C_{1}=-\frac{1}{2} \frac{16 b^{4} \lambda+a^{2}+2 b^{2}}{b}, \\
& C_{2}=-\frac{1}{2 b}\left(-\frac{\left(16 b^{4} \lambda+a^{2}+2 b^{2}\right) a}{b}+\frac{2 a^{3}}{b}-\frac{\left(-24 b^{4} \lambda+2 a^{2}-2 b^{2}\right) a}{b}-24 b^{3} a \lambda\right), \quad I_{0}=-\frac{a}{b}, \quad I_{1}=2 b, \quad m_{1}=-2 b \lambda
\end{aligned}
$$




\section{Set-7:}

$C_{1}=-\frac{1}{2} \frac{16 b^{4} \lambda+a^{2}+2 b^{2}}{b}$

$C_{2}=-\frac{1}{2 b}\left(-\frac{\left(16 b^{4} \lambda+a^{2}+2 b^{2}\right) a}{b}+\frac{2 a^{3}}{b}-\frac{\left(-24 b^{4} \lambda+2 a^{2}-2 b^{2}\right) a}{b}-24 b^{3} a \lambda\right), \quad I_{0}=-\frac{a}{b}, \quad I_{1}=-2 b, \quad m_{1}=2 b \lambda$

\section{Set-8:}

$C_{1}=-\frac{1}{2} \frac{-8 b^{4} \lambda+a^{2}+2 b^{2}}{b}$

$C_{2}=-\frac{1}{2 b}\left(-\frac{\left(-8 b^{4} \lambda+a^{2}+2 b^{2}\right) a}{b}+\frac{2 a^{3}}{b}-\frac{\left(24 b^{4} \lambda+2 a^{2}-2 b^{2}\right) a}{b}+24 b^{3} a \lambda\right), \quad I_{0}=-\frac{a}{b}, \quad I_{1}=-2 b, \quad m_{1}=-2 b \lambda$.

Assigning the Set-1 into the trial solution (12), we reach the results

(a) Hyperbolic function solutions (when $\lambda<0$ ):

$$
u_{16}(x, t)=-\frac{a}{b}-\frac{2 b\left[\sqrt{\left(I^{2}-d^{2}\right) \lambda}-I \sqrt{\lambda} \cos (2 \sqrt{\lambda}(\xi+E))\right]}{I \sin (2 \sqrt{\lambda}(\xi+E))+d},
$$

$u_{11}(x, t)=-\frac{a}{b}+\frac{2 b\left[\sqrt{-\left(I^{2}+d^{2}\right) \lambda}-I \sqrt{-\lambda} \cosh (2 \sqrt{-\lambda}(\xi+E))\right]}{I \sinh (2 \sqrt{-\lambda}(\xi+E))+d}$,

$u_{12}(x, t)=-\frac{a}{b}-\frac{2 b\left[\sqrt{-\left(I^{2}+d^{2}\right) \lambda}-I \sqrt{-\lambda} \cosh (2 \sqrt{-\lambda}(\xi+E))\right]}{I \sinh (2 \sqrt{-\lambda}(\xi+E))+d}$,

$u_{13}(x, t)=-\frac{a}{b}+\frac{2 b \sqrt{-\lambda}[\cosh (2 \sqrt{-\lambda}(\xi+E))-\sinh (2 \sqrt{-\lambda}(\xi+E))-I]}{I+\cosh (2 \sqrt{-\lambda}(\xi+E))-\sinh (2 \sqrt{-\lambda}(\xi+E))}$,

(b) Trigonometric function solutions (when $\lambda>0$ ):

$u_{14}(x, t)=-\frac{a}{b}+\frac{2 b \sqrt{-\lambda}[I-\cosh (2 \sqrt{-\lambda}(\xi+E))-\sinh (2 \sqrt{-\lambda}(\xi+E))]}{I+\cosh (2 \sqrt{-\lambda}(\xi+E))+\sinh (2 \sqrt{-\lambda}(\xi+E))}$,

$u_{15}(x, t)=-\frac{a}{b}+\frac{2 b\left[\sqrt{\left(I^{2}-d^{2}\right) \lambda}-I \sqrt{\lambda} \cos (2 \sqrt{\lambda}(\xi+E))\right]}{I \sin (2 \sqrt{\lambda}(\xi+E))+d}$,

$$
\begin{aligned}
& u_{17}(x, t)=-\frac{a}{b}+\frac{2 b \sqrt{\lambda}[i \cos (2 \sqrt{\lambda}(\xi+E))+\sin (2 \sqrt{\lambda}(\xi+E))-i l]}{1+\cos (2 \sqrt{\lambda}(\xi+E))-i \sin (2 \sqrt{\lambda}(\xi+E))} \\
& u_{18}(x, t)=-\frac{a}{b}+\frac{2 b \sqrt{\lambda}[-i \cos (2 \sqrt{\lambda}(\xi+E))+\sin (2 \sqrt{\lambda}(\xi+E))+i l]}{1+\cos (2 \sqrt{\lambda}(\xi+E))+i \sin (2 \sqrt{\lambda}(\xi+E))},
\end{aligned}
$$


(c) Rational function solutions (when $\lambda=0$ )

$u_{19}(x, t)=-\frac{a}{b}-\frac{2 b}{\xi+E}$

where $\xi=a \frac{t^{\alpha}}{\alpha}+b \frac{x^{\beta}}{\beta}$.

Assigning the Set-3 into the trial solution (12), we reach the results

(a) Hyperbolic function solutions (when $\lambda<0$ ):

$$
u_{35}(x, t)=-\frac{a}{b}+\frac{2 b[I \sin (2 \sqrt{\lambda}(\xi+E))+d]}{\sqrt{\left(I^{2}-d^{2}\right) \lambda}-I \sqrt{\lambda} \cos (2 \sqrt{\lambda}(\xi+E))}
$$

$$
u_{36}(x, t)=-\frac{a}{b}-\frac{2 b[I \sin (2 \sqrt{\lambda}(\xi+E))+d]}{\sqrt{\left(I^{2}-d^{2}\right) \lambda}-I \sqrt{\lambda} \cos (2 \sqrt{\lambda}(\xi+E))}
$$

$u_{31}(x, t)=-\frac{a}{b}+\frac{2 b[I \sinh (2 \sqrt{-\lambda}(\xi+E))+d]}{\sqrt{-\left(I^{2}+d^{2}\right) \lambda}-I \sqrt{-\lambda} \cosh (2 \sqrt{-\lambda}(\xi+E))}$,

$u_{32}(x, t)=-\frac{a}{b}-\frac{2 b[I \sinh (2 \sqrt{-\lambda}(\xi+E))+d]}{\sqrt{-\left(I^{2}+d^{2}\right) \lambda}-I \sqrt{-\lambda} \cosh (2 \sqrt{-\lambda}(\xi+E))}$,

$u_{33}(x, t)=-\frac{a}{b}+\frac{2 b \sqrt{-\lambda}[\cosh (2 \sqrt{-\lambda}(\xi+E))-\sinh (2 \sqrt{-\lambda}(\xi+E))-I]}{I+\cosh (2 \sqrt{-\lambda}(\xi+E))-\sinh (2 \sqrt{-\lambda}(\xi+E))}$,

(b) Trigonometric function solutions (when $\lambda>0$ ):

$u_{34}(x, t)=-\frac{a}{b}+\frac{2 b[I+\cosh (2 \sqrt{-\lambda}(\xi+E))+\sinh (2 \sqrt{-\lambda}(\xi+E))]}{\sqrt{-\lambda}[I-\cosh (2 \sqrt{-\lambda}(\xi+E))-\sinh (2 \sqrt{-\lambda}(\xi+E))]}$

Fig. 1 The profile of solution $u_{11}$ of the space-time FBK equation sketched for the parameters $I=a=E=1, b=0.5$ $\lambda=-2, a=\beta=3 / 4$ : (a) 3D plot; (b) $2 \mathrm{D}$ plots

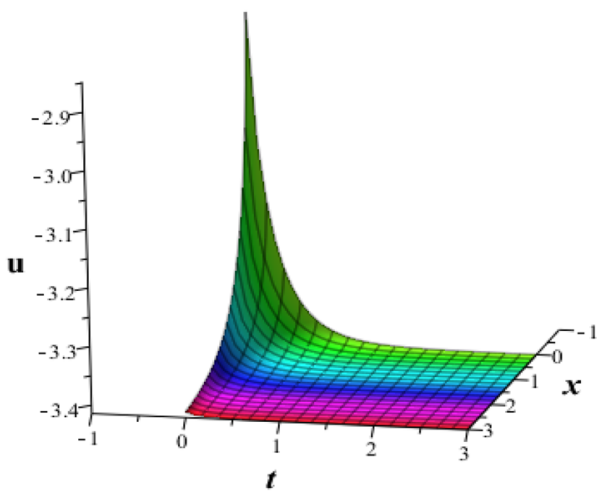

(a)

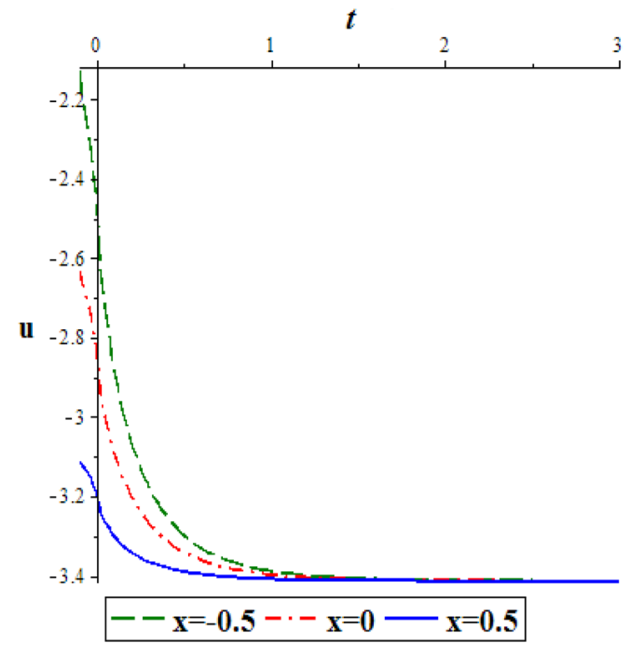

(b) 
Fig. 2 The profile of rogue into a periodic waves of the solution $u_{17}$ for the parameters $I=2, a=b=\lambda=1, a=\beta=3 / 4$, $E=0$ : (a) 3D (upper), contour (lower) plots of real part; (b) 3D (upper), contour (lower) plots of imaginary part; (c) 2D plots real part; (d) 2D plots of imaginary part

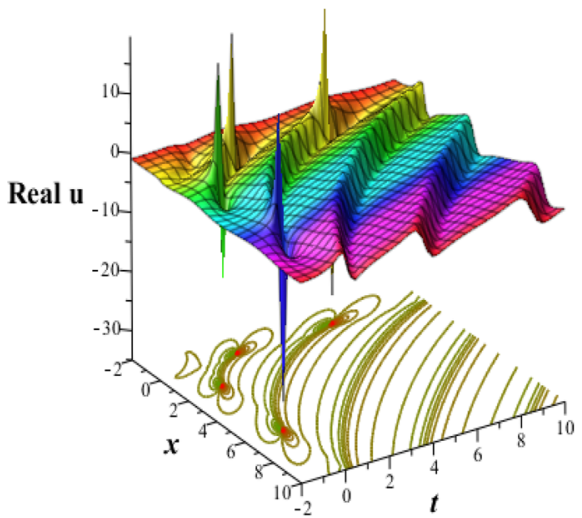

(a)

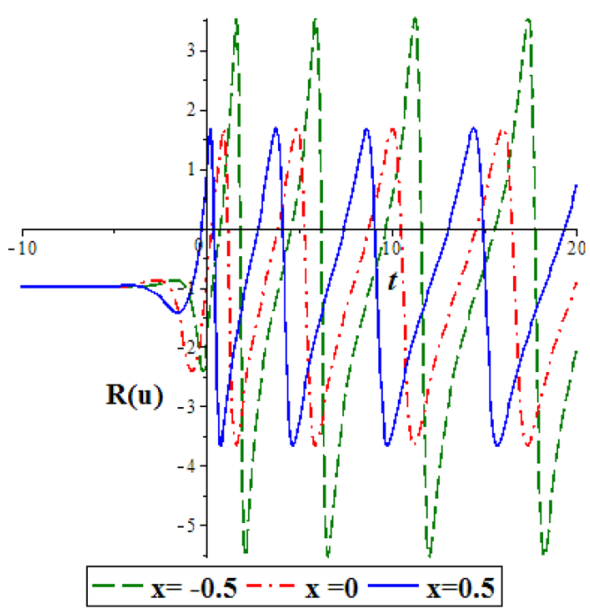

(c)

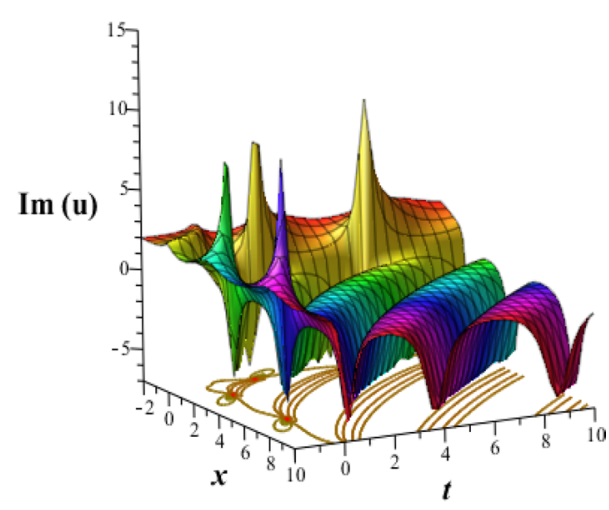

(b)

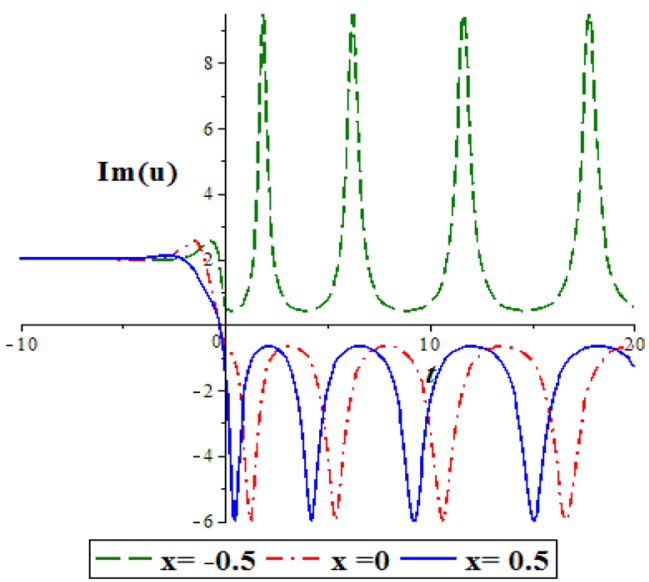

(d)

$u_{37}(x, t)=-\frac{a}{b}+\frac{2 b[I+\cos (2 \sqrt{\lambda}(\xi+E))-i \sin (2 \sqrt{\lambda}(\xi+E))]}{\sqrt{\lambda}[i \cos (2 \sqrt{\lambda}(\xi+E))+\sin (2 \sqrt{\lambda}(\xi+E))-i l]}$,

(c) Rational function solutions (when $\lambda=0$ )

$u_{38}(x, t)=-\frac{a}{b}+\frac{2 b[I+\cos (2 \sqrt{\lambda}(\xi+E))+i \sin (2 \sqrt{\lambda}(\xi+E))]}{\sqrt{\lambda}[-i \cos (2 \sqrt{\lambda}(\xi+E))+\sin (2 \sqrt{\lambda}(\xi+E))+i l]}$,

$u_{39}(x, t)=-\frac{a}{b}-2 b(\xi+E)$,

where $\xi=a \frac{t^{\alpha}}{\alpha}+b \frac{x^{\beta}}{\beta}$.

Assigning the Set-5 into the trial solution (12), we reach the results

(a) Hyperbolic function solutions (when $\lambda<0$ ): 
Fig. 3 The profile of rogue into a periodic waves of the solution $u_{18}$ for the parameters $I=2, a=b=\lambda=1, a=\beta=3 / 4$, $E=0$ : (a) 3D (upper), contour (lower) plots of real part; (b) 3D (upper), contour (lower) plots of imaginary part; (c) 2D plots real part; (d) 2D plots of imaginary part

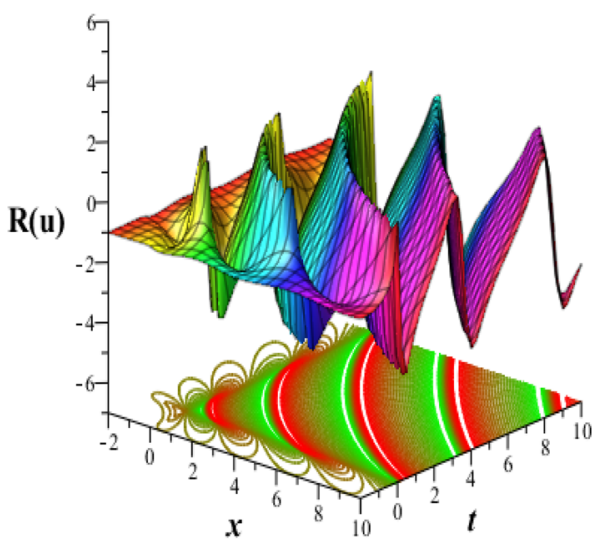

(a)

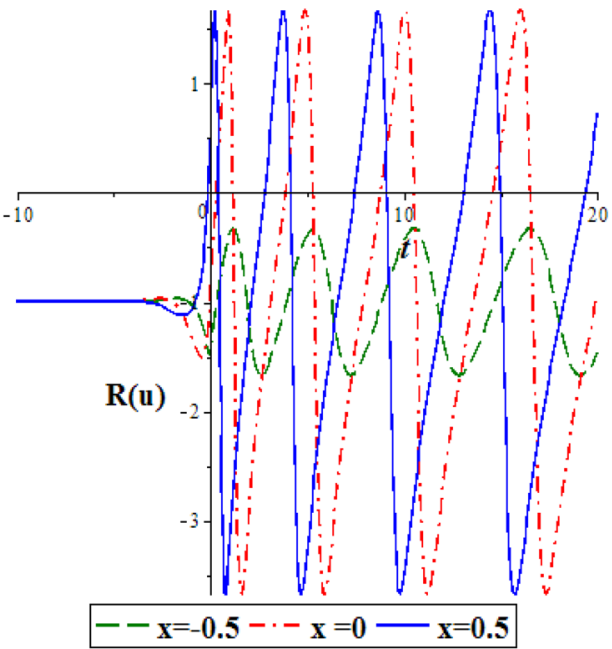

(c)

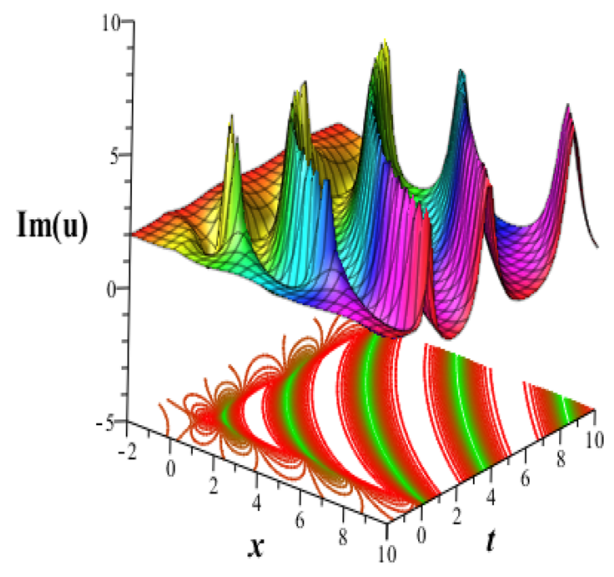

(b)

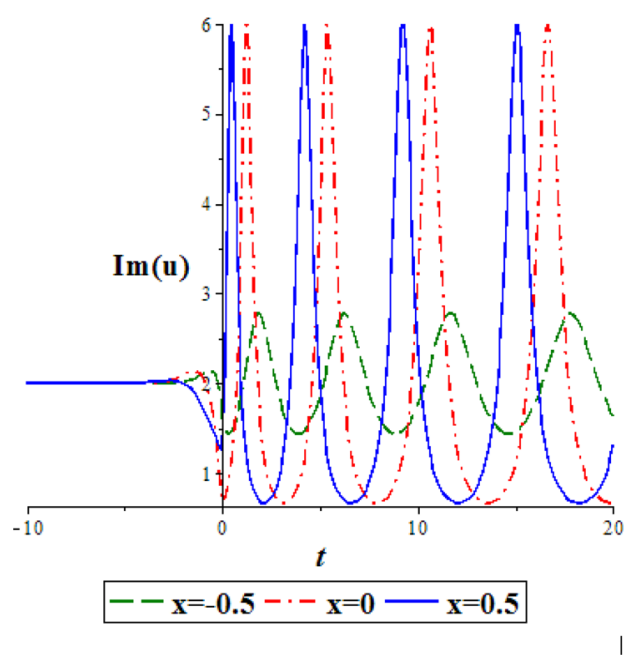

(d)
Fig. 4 The profile of solution $u_{19}$ of the space-time FBK equation sketched for the parameters $E=3, a=1, b=0.5$, $\lambda=0, a=\beta=1 / 2$ : (a) 3D plot; (b) 2D plots

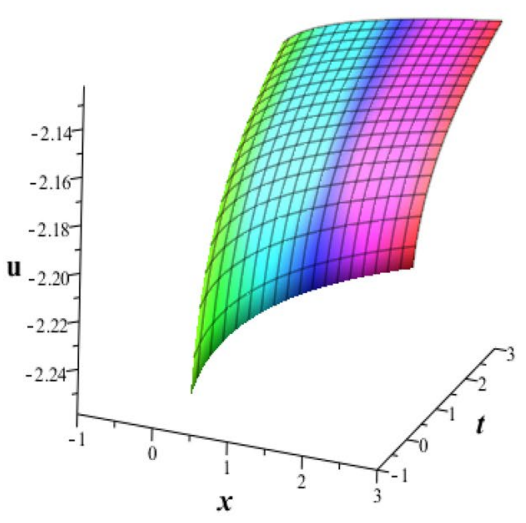

(a)

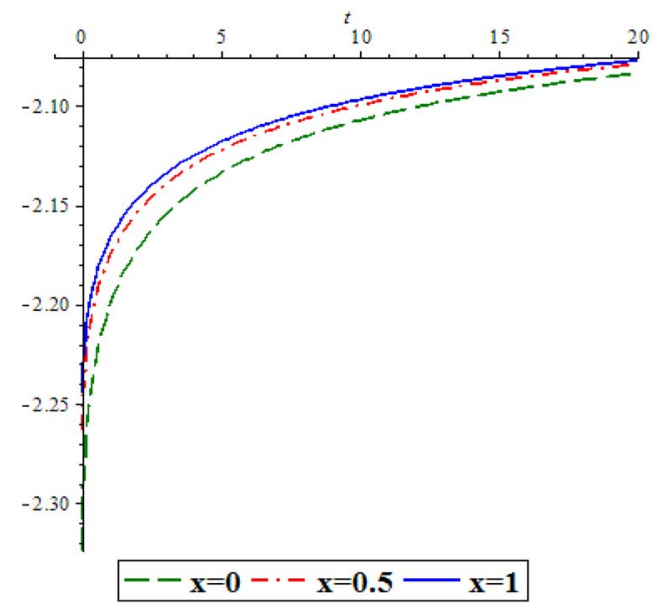

(b) 
$u_{51}(x, t)=-\frac{a}{b}+\frac{2 b\left[\sqrt{-\left(I^{2}+d^{2}\right) \lambda}-I \sqrt{-\lambda} \cosh (2 \sqrt{-\lambda}(\xi+E))\right]}{I \sinh (2 \sqrt{-\lambda}(\xi+E))+d}+\frac{2 b \lambda[I \sinh (2 \sqrt{-\lambda}(\xi+E))+d]}{\sqrt{-\left(I^{2}+d^{2}\right) \lambda}-I \sqrt{-\lambda} \cosh (2 \sqrt{-\lambda}(\xi+E))^{\prime}}$

$u_{52}(x, t)=-\frac{a}{b}-\frac{2 b\left[\sqrt{-\left(I^{2}+d^{2}\right) \lambda}-I \sqrt{-\lambda} \cosh (2 \sqrt{-\lambda}(\xi+E))\right]}{I \sinh (2 \sqrt{-\lambda}(\xi+E))+d}-\frac{2 b \lambda[I \sinh (2 \sqrt{-\lambda}(\xi+E))+d]}{\sqrt{-\left(I^{2}+d^{2}\right) \lambda}-I \sqrt{-\lambda} \cosh (2 \sqrt{-\lambda}(\xi+E))}$

$u_{53}(x, t)=-\frac{a}{b}+\frac{2 b \sqrt{-\lambda}[\cosh (2 \sqrt{-\lambda}(\xi+E))-\sinh (2 \sqrt{-\lambda}(\xi+E))-1]}{1+\cosh (2 \sqrt{-\lambda}(\xi+E))-\sinh (2 \sqrt{-\lambda}(\xi+E))}+\frac{2 b \lambda \sqrt{-\lambda}[\cosh (2 \sqrt{-\lambda}(\xi+E))-\sinh (2 \sqrt{-\lambda}(\xi+E))-1]}{1+\cosh (2 \sqrt{-\lambda}(\xi+E))-\sinh (2 \sqrt{-\lambda}(\xi+E))}$,

(b) Trigonometric function solutions (when $\lambda>0$ ):

$u_{54}(x, t)=-\frac{a}{b}+\frac{2 b \sqrt{-\lambda}[1-\cosh (2 \sqrt{-\lambda}(\xi+E))-\sinh (2 \sqrt{-\lambda}(\xi+E))]}{1+\cosh (2 \sqrt{-\lambda}(\xi+E))+\sinh (2 \sqrt{-\lambda}(\xi+E))}+\frac{2 b \lambda[1+\cosh (2 \sqrt{-\lambda}(\xi+E))+\sinh (2 \sqrt{-\lambda}(\xi+E))]}{\sqrt{-\lambda}[1-\cosh (2 \sqrt{-\lambda}(\xi+E))-\sinh (2 \sqrt{-\lambda}(\xi+E))]}$,

$u_{55}(x, t)=-\frac{a}{b}+\frac{2 b\left[\sqrt{\left(I^{2}-d^{2}\right) \lambda}-I \sqrt{\lambda} \cos (2 \sqrt{\lambda}(\xi+E))\right]}{I \sin (2 \sqrt{\lambda}(\xi+E))+d}+\frac{2 b \lambda[I \sin (2 \sqrt{\lambda}(\xi+E))+d]}{\sqrt{\left(I^{2}-d^{2}\right) \lambda}-I \sqrt{\lambda} \cos (2 \sqrt{\lambda}(\xi+E))}$,

$u_{56}(x, t)=-\frac{a}{b}-\frac{2 b\left[\sqrt{\left(I^{2}-d^{2}\right) \lambda}-I \sqrt{\lambda} \cos (2 \sqrt{\lambda}(\xi+E))\right]}{I \sin (2 \sqrt{\lambda}(\xi+E))+d}-\frac{2 b \lambda[I \sin (2 \sqrt{\lambda}(\xi+E))+d]}{\sqrt{\left(I^{2}-d^{2}\right) \lambda}-I \sqrt{\lambda} \cos (2 \sqrt{\lambda}(\xi+E))}$,

$u_{57}(x, t)=-\frac{a}{b}+\frac{2 b \sqrt{\lambda}[i \cos (2 \sqrt{\lambda}(\xi+E))+\sin (2 \sqrt{\lambda}(\xi+E))-i l]}{1+\cos (2 \sqrt{\lambda}(\xi+E))-i \sin (2 \sqrt{\lambda}(\xi+E))}+\frac{2 b \lambda[1+\cos (2 \sqrt{\lambda}(\xi+E))-i \sin (2 \sqrt{\lambda}(\xi+E))]}{\sqrt{\lambda}[i \cos (2 \sqrt{\lambda}(\xi+E))+\sin (2 \sqrt{\lambda}(\xi+E))-i l]}$,

(c) Rational function solutions (when $\lambda=0$ )

$u_{58}(x, t)=-\frac{a}{b}+\frac{2 b \sqrt{\lambda}[-i \cos (2 \sqrt{\lambda}(\xi+E))+\sin (2 \sqrt{\lambda}(\xi+E))+i l]}{1+\cos (2 \sqrt{\lambda}(\xi+E))+i \sin (2 \sqrt{\lambda}(\xi+E))}+\frac{2 b \lambda[1+\cos (2 \sqrt{\lambda}(\xi+E))+i \sin (2 \sqrt{\lambda}(\xi+E))]}{\sqrt{\lambda}[-i \cos (2 \sqrt{\lambda}(\xi+E))+\sin (2 \sqrt{\lambda}(\xi+E))+i l]}$, 
$u_{59}(x, t)=-\frac{a}{b}-\frac{2 b}{\xi+E}-2 b \lambda(\xi+E)$,

where $\xi=a \frac{t^{\alpha}}{\alpha}+b \frac{x^{\beta}}{\beta}$.

The other sets of solutions can obtain in the similar to the above three sets and we avoid these for simplicity.

Remark: The solutions obtained in this paper have been verified with Maple-18 by placing them back to the consider models (1).

\section{Clarification and graphical representations of the solutions}

In this segment, we discuss the physical exposition of securing solutions to the space-time FBKM by manipulating new vigorous method namely the unified method. We describe the behavior of all possible solutions with 3-D and 2-D plots. The solutions $u_{11}, u_{12}, u_{13}, u_{14}, u_{31}, u_{32}, u_{33}$, $u_{34}, u_{51}, u_{52}, u_{53}, u_{54}$ express hyperbolic function signifies solitonic nature. All the solutions have similar configurations and depicted only Fig. 1 of $u_{11}$.

From Fig. 1 we can see that the wave amplitudes abruptly change and go to infinity after reaching the beach of coaster area as $x, t \rightarrow 0$. The solutions $u_{15}, u_{16}$ $u_{17}, u_{18}, u_{35}, u_{36}, u_{37}, u_{38}, u_{55}, u_{56}, u_{57}, u_{58}$ express trigonometric function that have periodic behavior is depicted by Fig. 2 of $u_{17}$ only. From Fig. 2 we can see that the wave amplitudes remain unchanged in the deep ocean area (i.e. when $x, t$ are larger) but when waves reaches to shallow water in harbor region even in beach (i.e. when $x, t \rightarrow 0$ are going to smaller values), then wave amplitudes abruptly changes and some rogue type waves get into the periodic waves i.e. peaks and valleys arises into the each waves. We also provide the Fig. 3 of solution $u_{18}$ which has the similar behavior of Fig. 2 . The solutions $u_{19}, u_{39}, u_{59}$ express rational polynomial function with similar characteristics and we only plotted the figure of $u_{19}$ in Fig. 4. From Fig. 4 we can see that the wave amplitudes abruptly change to reduces after reaching the beach of coaster area as $x, t \rightarrow 0$.

\section{Comparison and discussion}

To best of our knowledge, only Yaslan [3] investigated the space-time model by using the $\exp (-\varphi(\xi))$-expansion method and obtained five solutions only (40), (41), (42), (43), (44) (See the Appendix). They got only five solutions including a tan, a tan hyperbolic, a combination of sine hyperbolic-cosine hyperbolic and two rational function solutions. Moreover, in this paper seventy-two solutions including combination of sine-cosine, combination of sine hyperbolic-cosine hyperbolic and rational function solutions for the space-time FBK are derived by applying unified method. But, by means of unified approach, we determined solutions which are different from Yaslan [3] solutions. We observed that their solutions (40), (41) and (44) are similar to our solutions $u_{11}, u_{15}$ and $u_{19}$ respectively (See the Appendix). Other solutions are completely new with more complex phenomena and were not obtained by Yaslan [3]. It is worth declaring that exp $(-\varphi(\xi))$ expansion method is special case of the unified method. Any researcher can compare their experimental/numerical results with our exact solutions to require actual investigation like Ref. [1, 2].

\section{Conclusion}

In this paper, we have fruitfully determined many new exact traveling wave solutions including combo periodicsolitary wave of the space-time FBKM by using the unified method. The dynamics of the obtained wave solutions are demonstrated in 2-D, 3-D and contour shapes. The Fig. 1 displayed that the wave amplitudes abruptly changed and gone to infinity after reaching the beach of coaster area as $x, t \rightarrow 0$, but the Fig. 4 showed that the wave amplitudes abruptly changed to reduces after reaching shallow water as $x, t \rightarrow 0$. Moreover, we have presented curvy periodic waves in which rogue waves occur in both sides of each waves (see Figs. 2 and 3). The researchers can compare their experimental/numerical results with our exact results like Ref. [1, 2], and civil engineer's may convey to predict disaster situations of harbor regions by taking preventive initiatives with help of our results. The future prospect of the fractional models holds strong. A lot of research lies ahead of the models such as study of conservation law, complexiton solutions, multi-soliton solutions and lie symmetry analysis etc.

\section{Compliance with ethical standards}

Conflict of interest The authors declare that they have no any conflict of interest.

\section{Appendix}

Yaslan [3] derived the exact solutions the space-time FBK model by using $\exp (-\varphi(\xi))$ expansion method. He derived the following five solutions:

For $\lambda^{2}-4 \mu>0, \mu \neq 0$, 
$u_{1}(x, t)=a_{0}+\frac{4 b \mu}{-\lambda-\sqrt{\lambda^{2}-4 \mu} \tanh \left[\frac{\sqrt{\lambda^{2}-4 \mu}}{2}\left\{b\left(-a_{0}+b \lambda\right) \frac{t^{\alpha}}{\alpha}+b \frac{x^{\beta}}{\beta}+C\right\}\right]}$,

For $\lambda^{2}-4 \mu<0, \mu \neq 0$,

$u_{2}(x, t)=a_{0}+\frac{4 b \mu}{-\lambda-\sqrt{4 \mu-\lambda^{2}} \tan \left[\frac{\sqrt{4 \mu-\lambda^{2}}}{2}\left\{b\left(-a_{0}+b \lambda\right) \frac{t^{\alpha}}{\alpha}+b \frac{x^{\beta}}{\beta}+C\right\}\right]}$,

For $\lambda^{2}-4 \mu>0, \mu=0, \lambda \neq 0$,

$u_{3}(x, t)=a_{0}+\frac{2 b \lambda}{\cosh \left[\left\{b\left(-a_{0}+b \lambda\right) \frac{t^{\alpha}}{\alpha}+b \frac{x^{\beta}}{\beta}+C\right\}\right]+\sinh \left[\left\{b\left(-a_{0}+b \lambda\right) \frac{t^{\alpha}}{\alpha}+b \frac{x^{\beta}}{\beta}+C\right\}\right]-1}$

For $\lambda^{2}-4 \mu=0, \mu \neq 0, \lambda \neq 0$,

$u_{4}=a_{0}-\frac{2 b \lambda^{2}\left[b\left(-a_{0}+b \lambda\right) \frac{t^{\alpha}}{\alpha}+b \frac{x^{\beta}}{\beta}+C\right]}{2 \lambda\left[b\left(-a_{0}+b \lambda\right) \frac{t^{\alpha}}{\alpha}+b \frac{x^{\beta}}{\beta}+C\right]+4}$

For $\lambda^{2}-4 \mu=0, \mu=0, \lambda=0$,

$u_{5}=a_{0}+\frac{2 b}{b\left(-a_{0}+b \lambda\right) \frac{t^{\alpha}}{\alpha}+b \frac{x^{\beta}}{\beta}+C}$.

\section{References}

1. Nistor I, Palermo D, Cornett A, Al-Faesly T (2010) Experimental and numerical modeling of tsunami loading on structures. In: Proceedings of the coastal engineering conference, 1-14. https ://doi.org/10.9753/icce.v32.currents.2.

2. Zaitsev Al, Kovalev DP, Kurkin AA, Levin BW, Pelinovsky EN, Chernov AG, Yalciner A (2008) The Nevelsk tsunami on August 2, 2007: Instrumental data and numerical modeling. Dokl Earth Sci 421(1):867-870. https://doi.org/10.1134/S1028334X080503 46

3. Yaslan HC (2018) New analytic solutions of the space-time fractional Broer-Kaup and approximate long water wave equations. J Ocean Eng Sci 3:295-302

4. Hammad MA, Khalil R (2014) Abel's formula and wronskian for conformable fractional differential equations. Int J Differ Equ Appl 13(3):177-183

5. Hirota R (1971) Exact solutions of the KdV equation for multiple collisions of solutions. Phys Rev Lett 27:1192-1194

6. Miura MR (1978) Backlund transformation. Springer, Berlin, Germany

7. Roshid HO (2017) Novel solitary wave solution in shallow water and ion acoustic plasma waves in-terms of two nonlinear models via MSE method. J Ocean Eng Sci 2(3):196-202

8. Wazwaz AM (2005) The tanh method: exact solutions of the Sine Gordon and the Sinh-Gordon equations. Appl Math Comput 49:565-574
9. Wazwaz AM (2004) The tanh method for travelling wave solutions of nonlinear equations. Appl Math Comput 154:713-723

10. Liu D (2005) Jacobi elliptic function solutions for variant Boussinesq equations. Chaos Soliton Fract 24:1373-1385

11. Akgül A, Kjijcman A, Inc M (2013) Improved ( $\left.G^{\prime} / G\right)$ Expansion method for the space and time fractional foam drainage and KdV equations. Abstr Appl Anal 2013:414353

12. Roshid HO, Alam MN, Hoque MF, Akbar MA (2013) A new extended $\left(G^{\prime} / G\right)$-expansion method to find exact traveling wave solutions of nonlinear evolution equations. Math Stat 1(3):162-166

13. Zayed EME, Abdelaziz MAM (2012) The two-variable $\left(\left(G^{\prime} / G\right)\right.$, $(1 / G)$ )-expansion method for solving the nonlinear KdV-mKdV equation. Math Probl Eng 2012:1-14

14. Bekir A (2008) Application of the $\left(G^{\prime} / G\right)$-expansion method for nonlinear evolution equations. Phys Lett A 372:3400-3406

15. L-Xiao L, E-Qiang L, M-Liang W (2010) The $\left(\left(G^{\prime} / G\right),(1 / G)\right)-$ expansion method and its application to travelling wave solutions of the Zakharov equations. Appl Math-A J Chinese Univ 25(4):454-462

16. Kudryashov NA (2009) On new travelling wave solutions of the $\mathrm{KdV}$ and KdV-Burgers equations. Commun Nonlinear Sci Numer Simul 14:1891-1900

17. Hossen MB, Roshid HO, Ali MZ (2017) Modified double subequation method for finding complexiton solutions to the $(2+1)$ dimensional nonlinear evolution equations. Int J Appl Comput Math 3(1):679-697

18. Roshid HO, Ma WX (2018) Dynamics of mixed lump-solitary waves of an extended (2+1)-dimensional shallow water wave model. Phys Lett A 382(45):3262-3268

19. Roshid HO, Khan MH, Wazwaz AM (2020) Lump, multi-lump, cross kinky-lump and manifold periodic-soliton solutions for the (2+1)-D Calogero_Bogoyavlenskii_Schiff equation. Heliyon 6:e03701

20. Guner O, Bekir A (2018) Solving nonlinear space-time fractional differential equations via ansatz method. Comput Methods Diff Equ 6(1):1-11 http://cmde.tabrizu.ac.ir

21. Güner Ö, Bekir A (2015) Exact solutions of some fractional differential equations arising in mathematical biology. Int J Biomath 8(1):1550003

22. Tozar A, Kurt A, Tasbozan O (2020) New wave solutions of time fractional integrable dispersive wave equation arising in ocean engineering models. Kuwait J Sci 47(2):22-33 
23. Kurt A (2020) New analytical and numerical results for fractional Bogoyavlensky-Konopelchenko equation arising in fluid dynamics. Appl Math J Chin Univ 35:101-112

24. Kurt A, Senol M, Tasbozan O, Chand M (2019) Two reliable methods for the solution of fractional coupled Burgers' equation arising as a model of poly-dispersive sedimentation. Appl Math Non Sci 4(2):523-534

25. Tasbozan O (2019) New analytical solutions for time fractional Benjamin-Ono equation arising internal waves in deep water. China Ocean Eng 33(5):593-600

26. Tasbozan O, Solen M, Kurt A, Ozkan O (2018) New solutions of fractional Drinfeld-Sokolov-Wilson system in shallow water waves. Ocean Eng 161(1):62-68
27. Iyiola OS, Tasbozan O, Kurt A, Cenesiz Y (2017) On the analytical solutions of the system of conformable time-fractional Robertson equations with 1-D diffusion. Chaos Soliton Fract 94(1):1-7

28. Cenesiz Y, Baleanu D, Kurt A, Tasbozan O (2017) New exact solutions of Burgers' type equations with conformable derivative. Waves Random Complex Media 27(1):103-116

29. Gozukizil OF, Akcagil S, Aydemir T (2016) Unification of all hyperbolic tangent function methods. Open Phys 14:524-541

30. Akcagil S, Aydemir T (2018) A new application of the unified method. NTMSCI 6(1):185-199

Publisher's Note Springer Nature remains neutral with regard to jurisdictional claims in published maps and institutional affiliations 